\title{
Research Article \\ Effect of Nanodiamonds on Structure and Durability of Polyethylene Oxide-Based Nanocomposites
}

\author{
Rossella Arrigo, Gabriele Ruisi, Rosalia Teresi, and Nadka Tzankova Dintcheva \\ Dipartimento di Ingegneria Civile, Ambientale, Aerospaziale, dei Materiali, Università degli Studi di Palermo, \\ Viale delle Scienze, Ed. 6, 90128 Palermo, Italy \\ Correspondence should be addressed to Rossella Arrigo; rossella.arrigo@unipa.it
}

Received 26 July 2016; Revised 24 October 2016; Accepted 27 October 2016

Academic Editor: R. Torrecillas

Copyright (C) 2016 Rossella Arrigo et al. This is an open access article distributed under the Creative Commons Attribution License, which permits unrestricted use, distribution, and reproduction in any medium, provided the original work is properly cited.

Polymer-based nanocomposites containing nanodiamonds (NDs) are attractive multifunctional materials with a growing range of applications. In this work, in the frame of developing completely biocompatible systems, nanocomposites based on polyethylene oxide (PEO) and different amount of NDs have been formulated through melt mixing and fully characterized. In particular, the reinforcement effect of NDs in PEO has been probed through tensile tests, and the rheological response of PEO-based nanocomposites as a function of the nanoparticles amount has been investigated and discussed. The obtained results show that the presence of well-distributed NDs strengthens the mechanical performance of the nanocomposites and brings about an increase of the PEO crystallinity, suggesting a strong adhesion between NDs and polymer matrix. Furthermore, as a result of NDs adding, alterations of the rheological behaviour of neat PEO can be noticed, as NDs are able to significantly influence the long-range dynamics of PEO chains. Besides, accelerated aging tests demonstrate that NDs show a remarkable protective ability against PEO photodegradation, due to their ability to attenuate efficiently UV radiation. The latter opens up new avenues for the use of NDs as multifunctional nanofillers for polymer-based nanocomposites with enhanced photooxidative resistance.

\section{Introduction}

In the last years, significant efforts have been addressed towards the formulation of high performance polymer-based nanocomposites, suitable for a wide range of applications [1, 2]. For this purpose, the potential of several kinds of nanoparticles, differing for chemical structure and dimensionality, has been exploited to enhance the mechanical, thermal, and electrical properties of polymeric matrices and to provide them with new functionalities [3-5]. Among different nanofillers, nanodiamonds particles (NDs) hold a special place [6]. NDs, usually produced by detonation techniques, are composed of nanoparticles with diameter of about $5 \mathrm{~nm}$ and consist of an inert diamond core, surrounded by a graphitic shell terminated by a large number of tailorable functional groups $[7,8]$. The internal diamond structure provides NDs with superior elastic modulus, high thermal conductivity, and electrical resistivity and chemical stability $[9,10]$. The surface functional groups, interacting with polymer chains, generate good adhesion between NDs and polymer matrix, improving the nanocomposite overall properties [11]. Moreover, the small size of NDs and their spherical shape result in a large and accessible external surface that maximizes the interactions with polymer matrix in which NDs are dispersed [12, 13]. However, due to their strong tendency to agglomerate, NDs, inserted in a polymer matrix, tend to form aggregates. The latter act as defects in the matrix, causing a worsening of the mechanical properties. In order to enhance the dispersion of NDs within polymer matrices, several approaches have been pursued, including high shear mixing and chemical functionalization of NDs surface [14-16]. For instance, to enhance the compatibility between polyethylene and NDs, alkyl groups of different length have been covalently linked to nanoparticles surface [17]. The so formulated nanocomposites show an increase of crystallinity with increasing NDs content and length of grafted alkyl chains, which corresponds to an enhancement of Young's modulus and hardness. Importantly, it has been shown that NDs are nontoxic and biocompatible 
$[18,19]$, which makes them excellent candidates for the formulations of advanced materials for biomedical applications [20]. Additionally, since NDs are able to strongly absorb UV radiation, they can be used to protect polymeric matrices from photoaging [21], although insufficient attention has been paid so far to the use of NDs as protective nanofillers against polymer photodegradation. Whit this in mind, in the frame of developing completely biocompatible polymerbased systems with enhanced photodegradation stability, in this work we prepared nanocomposites based on polyethylene oxide (PEO) and different amount of NDs, potentially applicable for the formulation of biomedical devices. The dispersion of NDs at microscale level and the effect of NDs on the rheological, mechanical, and crystallization behaviour of PEO have been evaluated and discussed in detail, also considering the influence of interactions between nanoparticles and host matrix. A special attention has been paid to the study of the photooxidative stability of the nanocomposites, in order to probe the effectiveness of NDs in the protection of polymeric matrix against photoinduced degradation.

\section{Experimental Part}

2.1. Materials. Polyethylene oxide (PEO) was purchased by Sigma-Aldrich. It has average molecular weight 100,000 g/mol, melting point $T_{m}=65^{\circ} \mathrm{C}$ (determinate by DSC), and density $1.13 \mathrm{~g} / \mathrm{mL}$ at $25^{\circ} \mathrm{C}$.

Nanodiamonds (NDs) with spherical shape were purchased by Sigma-Aldrich. According to supplier specifications, their average particle size is $<10 \mathrm{~nm}$ and the specific surface area (determinate by BET) is $278-335 \mathrm{~m}^{2} / \mathrm{g}$.

2.2. Nanocomposite Preparation. PEO/NDs nanocomposites have been formulated by melt compounding with a Brabender mixer at $80^{\circ} \mathrm{C}, 50 \mathrm{rpm}$ for 5 minutes. The mixture of PEO and NDs has manually premixed at room temperature until a homogeneous powder was obtained and subsequently fed into the mixer for melt compounding. PEO-based nanocomposites with $0.5,1,3$, and $5 \mathrm{wt} \%$ of NDs, corresponding to 0.16 , $0.33,0.99$, and 1.67 vol. $\%$, respectively, have been prepared. The nanocomposites have been named PEO/XNDs, where X is the NDs weight ratio in the PEO matrix. The specimens for the subsequent analyses and thin films (thickness less than $100 \mu \mathrm{m}$ ) have been prepared by compression moulding in a Carver laboratory hydraulic press. The pure PEO matrix, used as reference material, has been subjected to the same procedure.

2.3. Characterizations. The dispersion of NDs in PEO matrix has been observed by Scanning Electron Microscopy (SEM), performed on nitrogen fractured radial surfaces of the investigated samples with a Philips ESEM XL30 microscope.

Rheological tests were performed using a strain-controlled rotational rheometer (ARES G2 by TA Instruments) in parallel plate geometry (plate diameter $25 \mathrm{~mm}$ ). The complex viscosity $\left(\eta^{*}\right)$, storage $\left(G^{\prime}\right)$, and loss $\left(G^{\prime \prime}\right)$ moduli were measured performing frequency sweep tests at $T=80^{\circ} \mathrm{C}$ from $10^{-2}$ to $10^{2} \mathrm{rad} / \mathrm{s}$ at a maximum strain of $2 \%$. As proved by preliminary strain sweep experiments, such an amplitude is low enough to be in the linear viscoelastic regime. Besides, linear stress relaxation measurements were carried out submitting the samples to a single step strain $\gamma_{0}=1 \%$, and the shear stress evolution during time $\sigma(t)$ was measured to obtain the relaxation modulus $G(t)=\sigma(t) / \gamma_{0}$.

Crystallization behaviour of neat PEO and formulated nanocomposites has been investigated by differential scanning calorimetry (DSC), using a Perkin-Elmer DSC7 calorimeter. All experiments were performed under dry $\mathrm{N}_{2}$ gas using samples of around $10 \mathrm{mg}$ in $40 \mu \mathrm{L}$ sealed aluminium pans. Four calorimetric scans (two heating: $30-120^{\circ} \mathrm{C}$ and two cooling: $120-30^{\circ} \mathrm{C}$ ) were performed for each sample at scanning heating/cooling rate of $10^{\circ} \mathrm{C} / \mathrm{min}$.

The crystallinity degree $\left(X_{c}\right)$ is calculated using the formula:

$$
X_{c}(\%)=\frac{\Delta H_{m}}{\Delta H^{0}\left(1-W_{f}\right)},
$$

where $\Delta H_{m}$ is the heat of melt of sample, $\Delta H^{0}$ is the heat of fusion for $100 \%$ crystalline PEO (205 J/g) [22], and $W_{f}$ is the mass fraction of the nanofiller. The reported results are the average of three independent measurements.

Mechanical characterization was performed by using an Instron (USA) 3365 universal machine. The test was performed at two different speeds: $1 \mathrm{~mm} / \mathrm{min}$ for the first $2 \mathrm{~mm}$ of elongation and then $100 \mathrm{~mm} / \mathrm{min}$ up to sample breakage. The average values for elastic modulus, tensile strength, and elongation at break were calculated and the standard deviation is reported in the figures.

The photooxidative resistance of the materials was estimated using a Q-UV-Solar Eye weatherometer (Q-LAB, USA) equipped with UVB lamps $(313 \mathrm{~nm})$. The tests were carried out on the thin films. The weathering conditions were 24 h of light at $T=45^{\circ} \mathrm{C}$, in order to operate below the melting point of PEO. The progress of the photodegradation was followed by analysing the evolution in time of FTIR spectra on polymer films carried out by using a Perkin-Elmer FTIR spectrometer (mod. Spectrum Two). FTIR spectra collected on three different films of each sample were obtained by performing 16 scans between 4000 and $500 \mathrm{~cm}^{-1}$. The photooxidation evolution was quantified by referring to the Carbonyl Index (CI) as a function of irradiation time. CI was calculated as the ratio between the integral of the carbonyl absorption region $\left(1850-1600 \mathrm{~cm}^{-1}\right)$ and that of a reference peak at about $1965 \mathrm{~cm}^{-1}$, which account for the sample thickness variation [23].

\section{Results and Discussion}

3.1. Characterization of PEO/NDs Nanocomposites. First of all, the internal structure of NDs-containing nanocomposites has been observed by means of SEM, which allows us to evaluate the nanoparticles dispersion as well as the compatibility between the nanofillers and the host polymer matrix. The evaluation of the dispersion state of the nanoparticles in a polymer matrix is crucial since it plays a key role in enhancing the final properties of resulting nanocomposites. In Figure 1 the SEM micrographs at low and high magnification for 


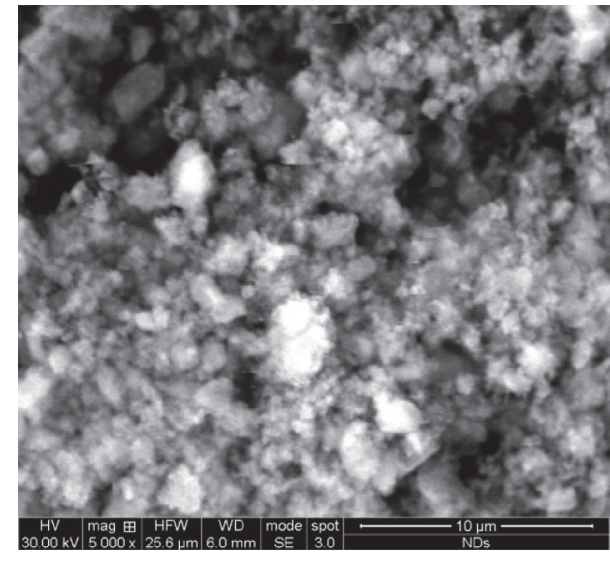

Pristine NDs

(a)

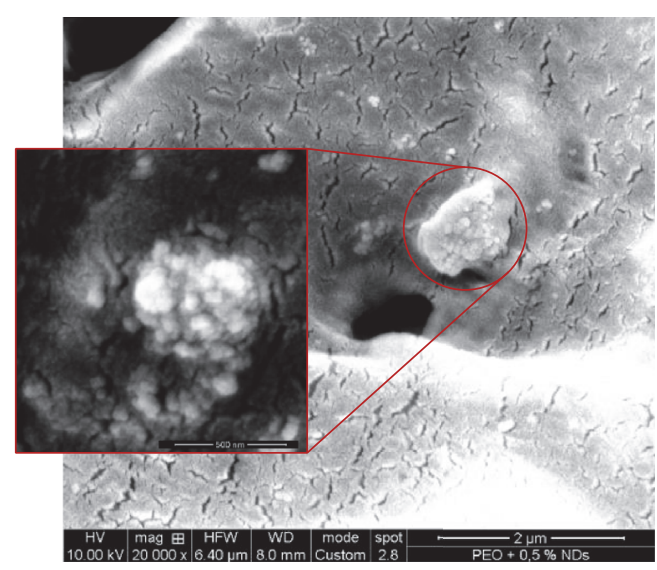

PEO/0.5NDs

(c)

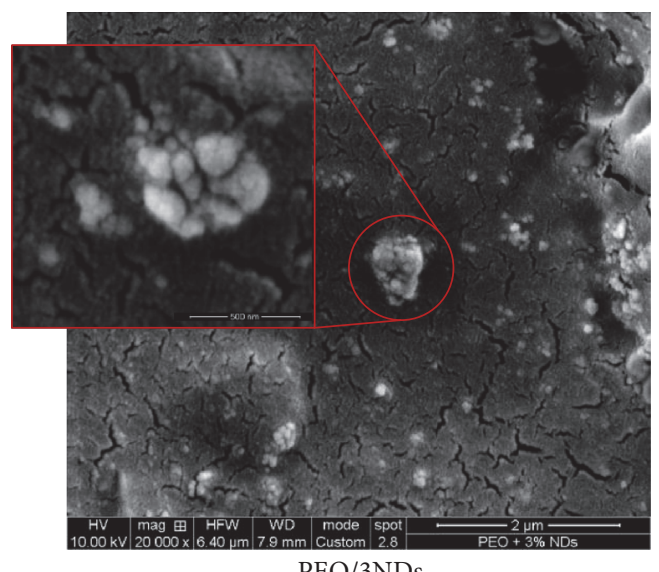

PEO/3NDs

(e)

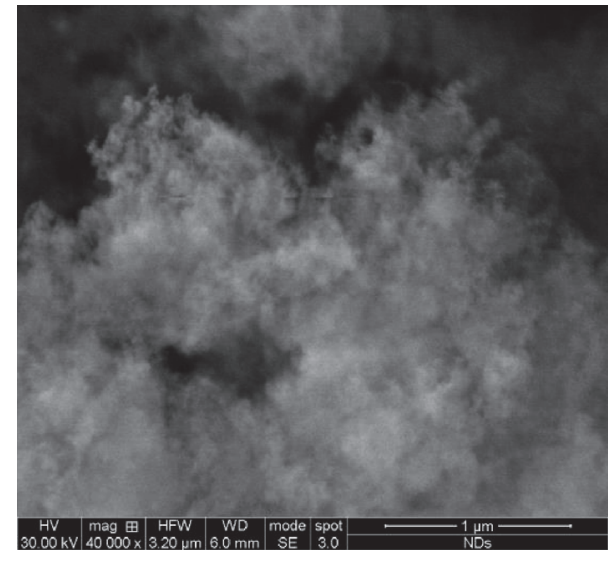

Pristine NDs

(b)

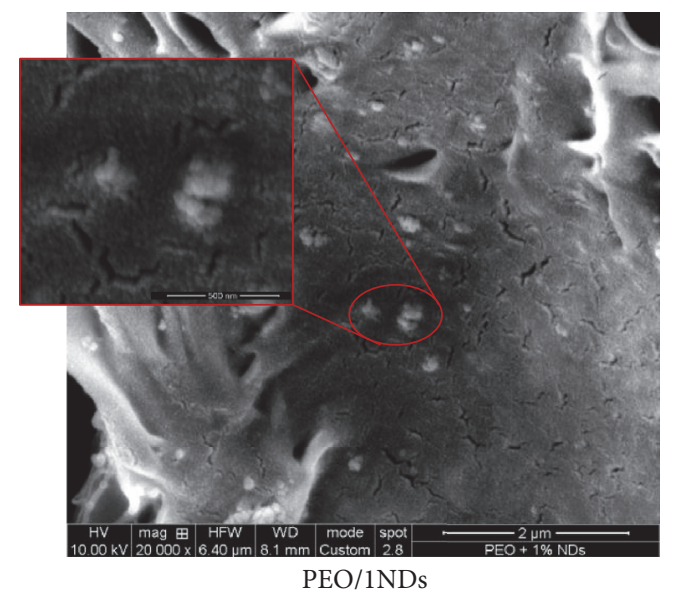

(d)

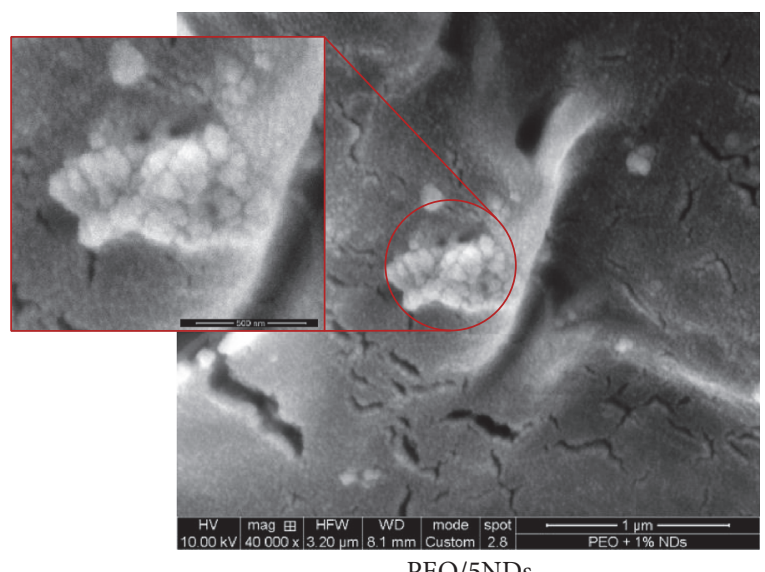

(f)

FIGURE 1: SEM observations at different magnifications of pristine NDs and all NDs-containing nanocomposites. In the insets, SEM micrographs at high magnification $(80000 \mathrm{x})$ are reported. 


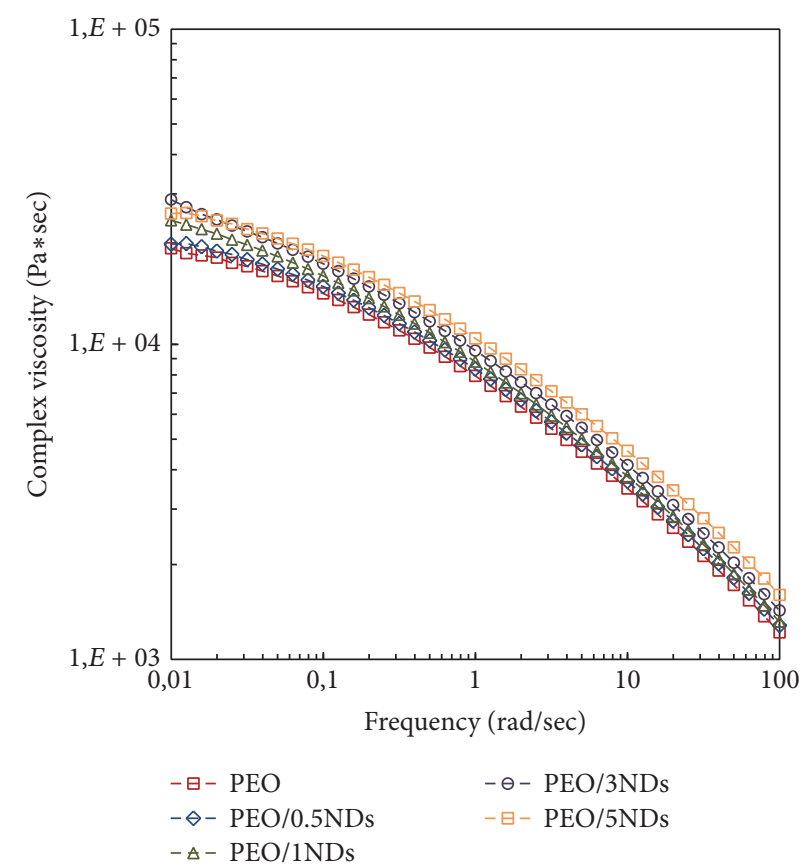

(a)

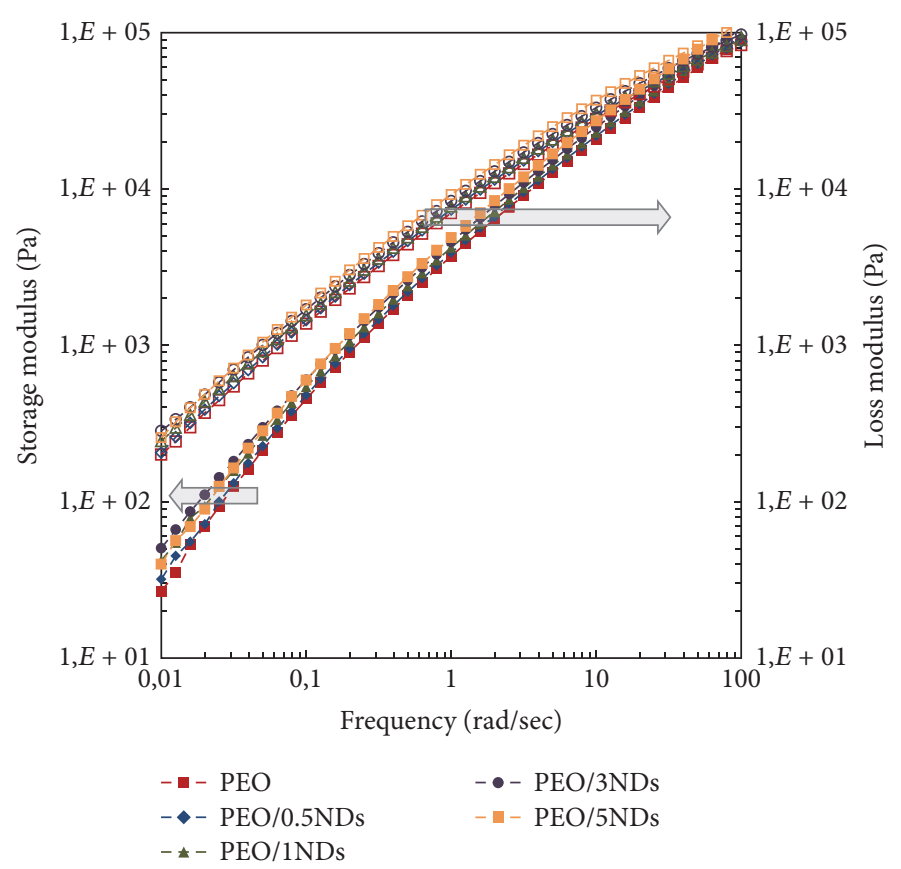

(b)

FIGURE 2: Complex viscosity (a) and storage and loss moduli (b) as a function of frequency for neat PEO and PEO-based nanocomposites.

pristine NDs and PEO-based nanocomposites are shown. It is well known that pristine NDs tend to form aggregates and agglomerates because of their high surface energy and the presence of several functional groups on their surface; see Figures 1(a) and 1(b). Concerning PEO-based nanocomposites, NDs in the form of nanoclusters are uniformly and homogeneously dispersed in the host polymer matrix. The average dimension of formed agglomerates is about $300 \mathrm{~nm}$, as visible in micrographs at high magnification, and no increase in agglomerates size and density can be observed up to $3 \mathrm{wt} . \%$. of NDs content; differently, as the amount of NDs increases, the presence of some coarse agglomerate can be noticed.

The obtained good dispersion state of NDs nanoclusters can be ascribed, first, to the appropriate conditions, such as temperature and mixing time, used for the nanocomposites processing. Furthermore, it is reasonable to suppose that the functional groups onto NDs surface can interact with functional groups of PEO matrix and that the established interactions can promote the obtained uniform dispersion of NDs nanoclusters within host matrix.

Rheological properties can provide useful information about the interactions between filler and polymer matrix in polymer-based nanocomposites and the state of dispersion and distribution of nanofillers. The complex viscosity $\left(\eta^{*}\right)$ curves of neat $\mathrm{PEO}$ and PEO-based nanocomposites are shown in Figure 2(a) as a function of frequency. At low frequencies, neat matrix exhibits the typical Newtonian viscosity plateau, due to the fully relaxed PEO chains; as frequency increases, PEO macromolecular chains start to align with the flow and a shear thinning behaviour can be noticed. The adding of $0.5 \mathrm{wt} . \%$ of NDs does not significantly affect the rheological behaviour of PEO; indeed the values of $\eta^{*}$ for
$\mathrm{PEO} / 0.5 \mathrm{NDs}$ nanocomposite are very similar to those of neat PEO, and the Newtonian plateau is still present. Differently, as the NDs content increases up to $3 \mathrm{wt} . \%$, the viscosity values of nanocomposites are higher than those of neat PEO in the whole investigated frequency range, particularly at low frequencies, and the disappearance of the Newtonian behaviour occurs. At high frequencies, the effect of NDs on PEO rheological behaviour is relatively weak, suggesting that NDs do not significantly influence the short-range dynamics of PEO chains, particularly on length scales comparable to the matrix entanglements length. Concerning the nanocomposite $\mathrm{PEO} / 5 \mathrm{NDs}$, a mere vertical shift of the $\eta^{*}$ curve toward higher values with respect to neat PEO can be noticed. In other words, the adding of $5 \mathrm{wt} . \%$ of NDs causes an increase of the viscosity values, but the frequency-dependence of the $\eta^{*}$ remains unaltered compared to the neat matrix. The latter can be understood considering that the NDs aggregates in this sample are too large to generate appreciable rheological alterations and the rheological response of the nanocomposite is governed by rheological behaviour of PEO.

The storage $\left(G^{\prime}\right)$ and loss $\left(G^{\prime \prime}\right)$ moduli as a function of the frequency for neat PEO and NDs-containing nanocomposites are reported in Figure 2(b). Consistent with the addition of solid particles into a polymer matrix, both moduli of PEO-based systems increase at all frequencies with the NDs adding, following the same trend observed for the viscosity. Moreover, the terminal behaviour tends to disappear for nanocomposites containing high amount of NDs, and the dependence of both $G^{\prime}$ and $G^{\prime \prime}$ on frequency at low frequency becomes weak. Generally, the modification of polymer rheological behaviour due to the adding of solid nanoparticles is attributed to the establishment of two kinds of interactions: 


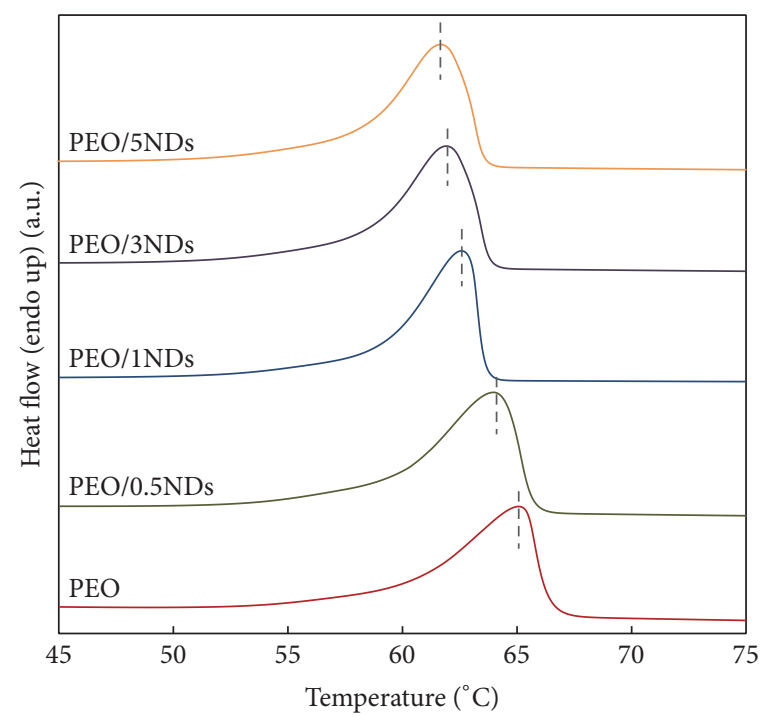

FIGURE 3: DSC thermograms recorded during second heating scan for neat PEO and all NDs-containing nanocomposites.

TABLE 1: Thermal properties of neat PEO and all investigated systems evaluated during second heating scan.

\begin{tabular}{lccc}
\hline Sample & $T_{\text {melt }}\left[{ }^{\circ} \mathrm{C}\right]$ & $\Delta H_{\text {melt }}[\mathrm{J} / \mathrm{g}]$ & $X_{c}[\%]$ \\
\hline PEO & 65.0 & 135.4 & 66 \\
PEO/0.5NDs & 64.0 & 136.2 & 66.8 \\
PEO/1NDs & 62.7 & 136.8 & 67.4 \\
PEO/3NDs & 62.2 & 137.5 & 69.1 \\
PEO/5NDs & 61.8 & 137.4 & 70.5 \\
\hline
\end{tabular}

particle-polymer interaction and particle-particle interaction. The former can be expressed in terms of absorption of polymer chains onto nanoparticles surface; specifically, the enhancement of viscosity values is believed to be due to an immobilized hydrodynamic layer near the nanoparticle with a thickness on the order of the radius of gyration of the polymer chain [24]. The particle-particle interactions, instead, play a predominant role in the rheological response of polymer-based nanocomposites $[25,26]$ and lead to the formation of a nanofiller network in the host matrix.

To assess the influence of NDs adding on the crystallization behaviour of PEO, DSC analyses have been carried out. In Figure 3, the DSC thermograms recorded during the second heating scan after fast cooling from the molten state (performed to erase the sample thermal history) are reported, and the relevant data, such as melting temperature and enthalpy and the degree of crystallinity, are listed in Table 1. It can be noticed that the introduction of NDs in PEO matrix results in a lowering of melting temperature from $65^{\circ} \mathrm{C}$ to $61.8^{\circ} \mathrm{C}$ for neat $\mathrm{PEO}$ and nanocomposite containing $5 \mathrm{wt} . \% \mathrm{NDs}$, respectively, and in an increase of melting enthalpy. The latter implies that the crystallinity of NDs-containing nanocomposites is higher than that of neat PEO. The enhancement of the crystallinity degree can be attributed to the nucleating action of NDs that promotes the formation of crystalline phase in PEO.

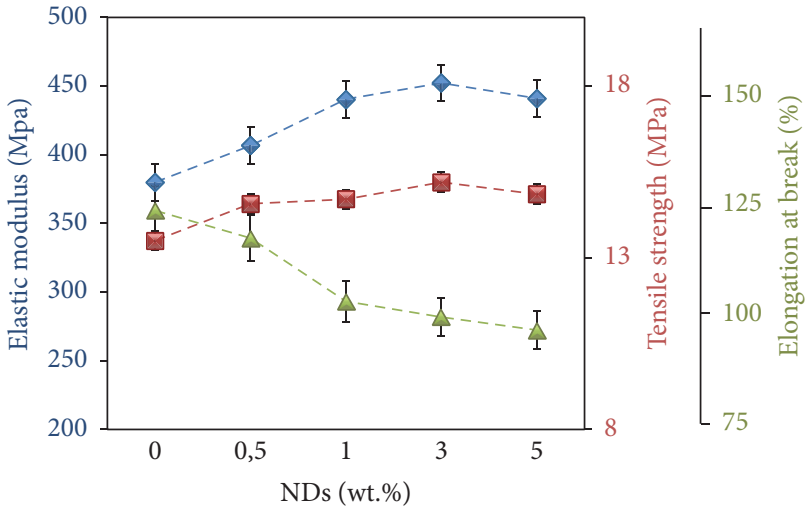

FIGURE 4: Main mechanical properties of neat PEO and all NDscontaining nanocomposites as a function of NDs content (the dashed lines are guide for the eye).

The mechanical behaviour of PEO and PEO-based nanocomposites has been evaluated through tensile tests. In Figure 4 , the main mechanical properties, such as elastic modulus (E), tensile strength (TS), and elongation at break (EB), as a function of NDs content are reported. Let us now consider the nanocomposites with NDs content in the range 0.5$3 \mathrm{wt} \%$. It is evident that the adding of NDs into PEO matrix leads to the enhancement of both E and TS at all concentrations studied. Differently, EB slightly decreases, indicating that the nanocomposites become somewhat brittle compared with neat PEO. Therefore, the tensile test results overall indicate that the mechanical properties of NDs-containing nanocomposites are substantially superior to those of neat PEO. The improvement of PEO mechanical properties can be ascribed, mainly, to the uniform dispersion of nanofillers throughout the host matrix. Indeed, it is known that the mechanical behaviour of a nanocomposite depends on the dispersion state of nanofillers in the matrix; morphological 


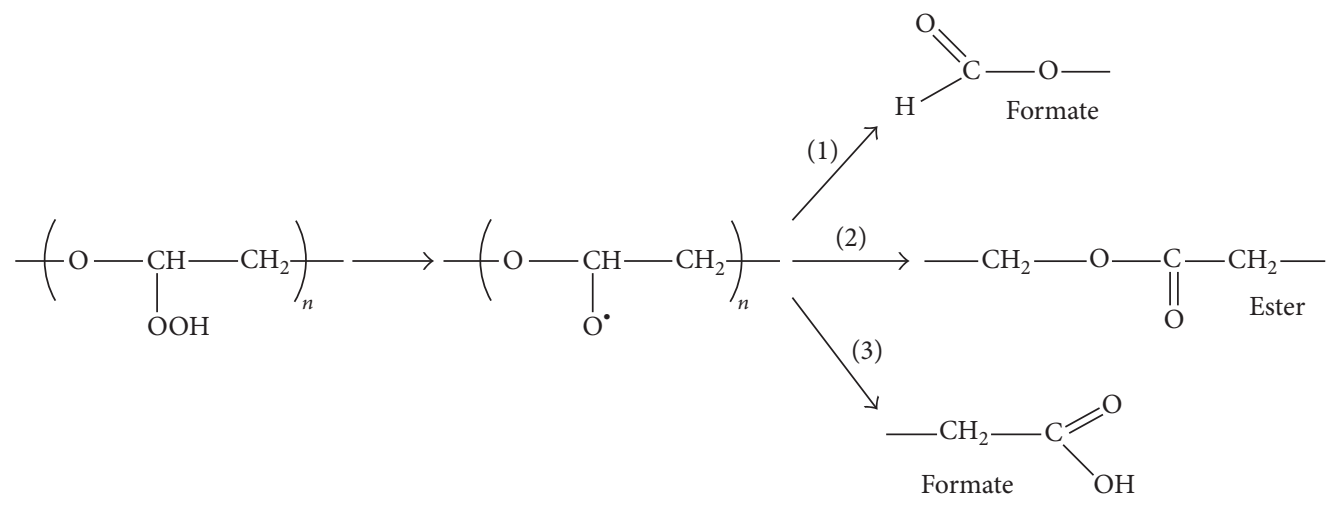

Figure 5: Photooxidation pathway of PEO [23].

analyses of PEO-based nanocomposites showed that NDs in the form of nanoclusters are distributed homogeneously within polymer matrix, and this issue is essential to obtain high performance materials. Also the characteristic of the interface between the reinforcing filler and the matrix plays a crucial role in determining the mechanical properties of a nanocomposite. Results coming from DSC analyses demonstrated an enhancement of crystallinity degree of nanocomposites with respect to neat $\mathrm{PEO}$, and this implies that the adhesion between NDs and matrix is strong, promoting the crystallization of PEO, which in turn enhances the mechanical properties of nanocomposites. Furthermore, the establishment of strong interaction between NDs and PEO promotes the stress transfer from nanoparticles to polymer matrix, strengthening the overall mechanical performance of the nanocomposites.

As far as the $\mathrm{PEO} / 5 \mathrm{NDs}$ nanocomposite is concerned, the tensile properties tend to decrease with respect to nanocomposites containing lesser amount of NDs, due to the poor dispersion of nanofillers achieved in this sample. Indeed, the large NDs aggregates act as defects in the nanocomposite structure and, according to the literature, cause a worsening of the nanocomposite mechanical behaviour [11].

\subsection{Photooxidation Behaviour of PEO/NDs Nanocomposites.} To investigate the photooxidation behaviour of PEO-based nanocomposites, thin films have been subjected to accelerate UVB exposure and the formation of oxidized species coming from degradation reactions as a function of the exposure time has been monitored through FTIR analysis. Although PEO does not absorb radiation of wavelengths longer than $300 \mathrm{~nm}$, it is very susceptible of photooxidation, due to chromophoric impurities which, absorbing UV light, produce radicals that react with polymer [27]. The first step of the oxidative pathway is the hydrogen abstraction on the polymer backbone by a free radical, with the formation of a macroradical that, reacting with oxygen, gives a peroxy radical that evolves in a hydroperoxide; see Figure 5. The photochemical decomposition of this hydroperoxide leads to the formation of an alkoxy radical that can evolve following three routes: (1) $\beta$-scission, leading to the formation of formate end groups; (2) cage reaction with $\mathrm{HO}^{\circ}$ which gives ester functions formation; (3) hydrogen abstraction reaction with formation of a hemiacetal that, in turn, decomposes to form alcohols and carboxylic acids [23]. Therefore, the main oxidation products of PEO formed upon UVB irradiation are essentially formats, esters, carboxylic acids, and alcohols, where accumulation can be evaluated analysing the evolution of FTIR spectra. In Figure 6(a), the carbonyl region of FTIR spectra collected during the photooxidative aging of neat PEO are shown. As the photooxidation time increases, the appearance of new absorption bands can be noticed: a band with an absorption maximum at $1725 \mathrm{~cm}^{-1}$ and a shoulder around $1750 \mathrm{~cm}^{-1}$. These new peaks can be attributed to the formation of formats and esters groups, respectively. Looking at FTIR spectra reported in Figures 6(b)-6(d), it is evident that the accumulation of oxidized species is lower for NDs-containing nanocomposites in comparison to the pristine $\mathrm{PEO}$, suggesting an unequivocal beneficial effect of the nanoparticles presence in the protection of PEO against photooxidative degradation. To evaluate the formation of the degradation species as a function of exposure time, the Carbonyl Index for all investigated systems has been calculated and plotted in Figure 7. The neat PEO experiences oxidative process since the early stage of UVB irradiation, due to the rapid formation of oxidized species, and no induction period can be detected. A dramatic increase of the photooxidative resistance characterizes the nanocomposites containing the NDs. Indeed, the values of Carbonyl Index for nanocomposites are definitely lower than those of neat PEO and, after a slight increase during the first $100 \mathrm{~h}$ of exposure, they remain constant over time. No significant differences due to the different content of NDs can be observed and the stabilization activity of NDs in PEO seems to be almost concentration-independent. Similar results have been reported by Ammala et al. [28] in the case of polyolefins-based nanocomposites containing zinc oxide $(\mathrm{ZnO})$ nanoparticles. The latter act as UV stabilizers for polypropylene and high density polyethylene and the obtained values of Carbonyl Index for nanocomposites containing 1 and $2 \mathrm{wt} \%$ of $\mathrm{ZnO}$ after about 1000 hours of photoaging are unaffected by the content of nanoparticles. In our case, the adding of $0.5 \mathrm{wt} \%$ of NDs results in a dramatic increase of the photooxidative resistance of PEO-based nanocomposites; 


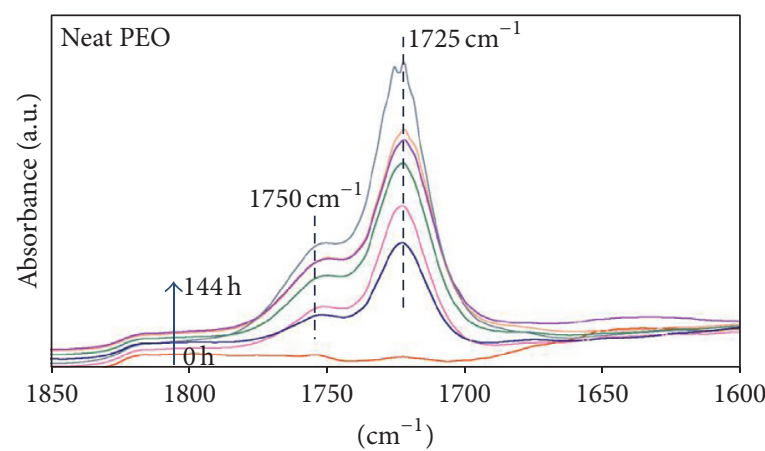

(a)

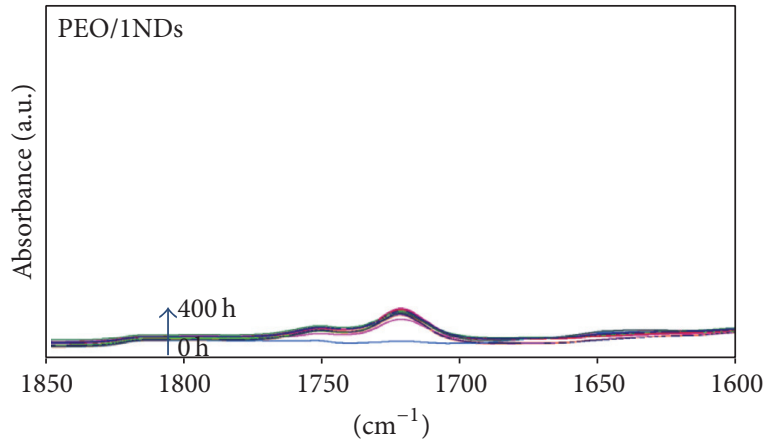

(c)

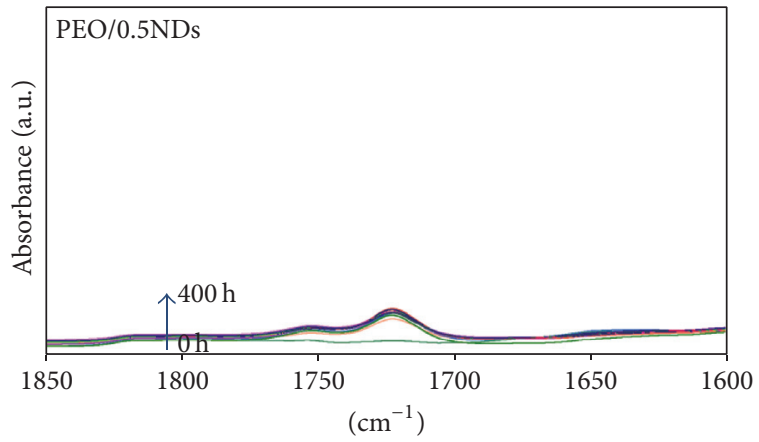

(b)

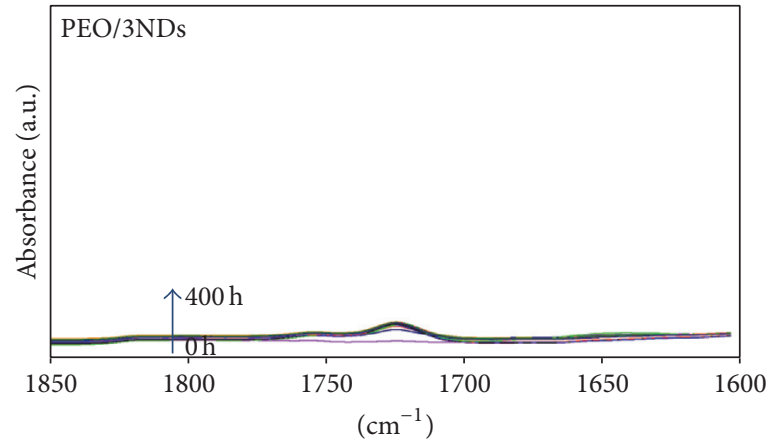

(d)

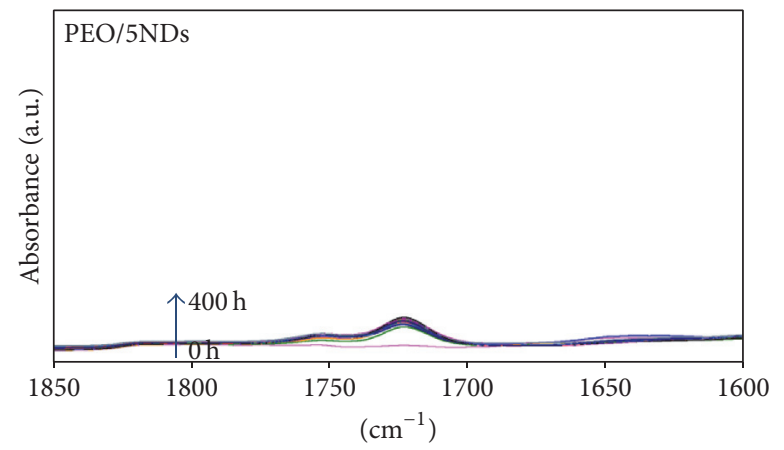

(e)

FIGURE 6: FTIR spectra in the carbonyl absorption region $\left(1850-1600 \mathrm{~cm}^{-1}\right)$ as a function of photooxidation time for all investigated systems.

any additional increase in the content of NDs does not result in a significant increase of the photooxidative stability.

NDs nanoparticles, thus, are able to significantly slow down the PEO degradation processes, exerting a remarkable stabilizing action against photooxidation. The latter can be understood considering that NDs, having a wide band gap $(5 \mathrm{eV})$, are highly absorptive toward UV light [21]. Furthermore, NDs produced by detonation consist of an $\mathrm{sp}^{3}$ carbon core, surrounded by a shell of $\mathrm{sp}^{2}$ carbon [29]. The graphitic carbon layer present on the external shell of NDs nanoparticles is responsible for the attenuation of UV radiation by NDs.

\section{Conclusions}

Nanocomposites based on PEO and different amount of NDs have been formulated and fully characterized, with the aim to develop fully biocompatible polymer-based systems potentially suitable for biomedical applications. Morphological analyses show that NDs, in the form of nanoclusters, are uniformly dispersed in the host matrix, and no increase in agglomerates density has been observed with increasing of NDs content up to $3 \mathrm{wt} \%$. Further, the occurrence of strong interactions between nanoparticles and polymer matrix and the obtainment of a good interfacial adhesion cause the increase of PEO crystallinity and the significant improvement of the mechanical properties of nanocomposites. Moreover, $\mathrm{PEO} / \mathrm{ND}$ nanocomposites exhibit enhanced photooxidative stability with respect to the neat matrix, due to the ability of NDs to attenuate efficiently the UV radiation. Therefore, NDs can be considered as promising nanofillers for high performance polymer-based nanocomposites with enhanced photooxidative resistance and prolonged durability. 


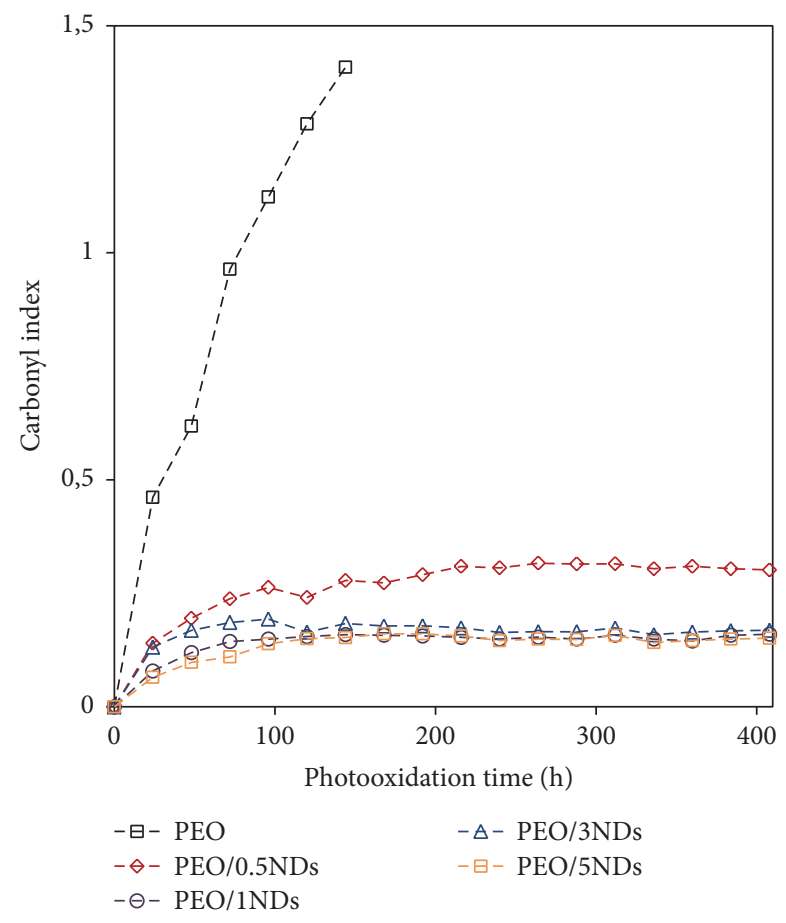

FIgURE 7: Carbonyl Index as a function of photooxidation time for neat $\mathrm{PEO}$ and NDs-containing nanocomposites.

\section{Competing Interests}

The authors declare that there is no conflict of interests regarding the publication of this paper.

\section{References}

[1] F. Navarro-Pardo, A. L. Martinez-Hernandez, and C. VelascoSantos, "Carbon nanotube and graphene based polyamide electrospun nanocomposites: a review," Journal of Nanomaterials, vol. 2016, Article ID 3182761, 16 pages, 2016.

[2] S. Kango, S. Kalia, A. Celli, J. Njuguna, Y. Habibi, and R. Kumar, "Surface modification of inorganic nanoparticles for development of organic-inorganic nanocomposites-a review," Progress in Polymer Science, vol. 38, no. 8, pp. 1232-1261, 2013.

[3] N. T. Dintcheva, R. Arrigo, E. Morici et al., "Multi-functional hindered amine light stabilizers-functionalized carbon nanotubes for advanced ultra-high molecular weight Polyethylenebased nanocomposites," Composites Part B: Engineering, vol. 82, pp. 196-204, 2015.

[4] A. Tesarikova, D. Merinska, J. Kalous, and P. Svoboda, "Ethylene-octene copolymers/organoclay nanocomposites: preparation and properties," Journal of Nanomaterials, vol. 2016, Article ID 6014064, 13 pages, 2016.

[5] H. Xie and W. Shi, "Polymer $/ \mathrm{SiO}_{2}$ hybrid nanocomposites prepared through the photoinitiator-free UV curing and sol-gel processes," Composites Science and Technology, vol. 93, pp. 9096, 2014.

[6] V. N. Mochalin and Y. Gogotsi, "Nanodiamond-polymer composites," Diamond and Related Materials, vol. 58, pp. 161-171, 2015.

[7] A. Shakun, J. Vuorinen, M. Hoikkanen, M. Poikelispää, and A. Das, "Hard nanodiamonds in soft rubbers: past, present and future-a review," Composites Part A: Applied Science and Manufacturing, vol. 64, pp. 49-69, 2014.

[8] U. Maitra, K. E. Prasad, U. Ramamurty, and C. N. R. Rao, "Mechanical properties of nanodiamond-reinforced polymermatrix composites," Solid State Communications, vol. 149, no. 39-40, pp. 1693-1697, 2009.

[9] A. Krueger, "The structure and reactivity of nanoscale diamond," Journal of Materials Chemistry, vol. 18, no. 13, pp. 14851492, 2008.

[10] V. Y. Dolmatov, "Detonation synthesis ultradispersed diamonds: properties and applications," Russian Chemical Reviews, vol. 70, no. 7, pp. 607-626, 2001.

[11] Y.-Q. Zhao, K.-T. Lau, J.-K. Kim, C.-L. Xu, D.-D. Zhao, and H.-L. $\mathrm{Li}$, "Nanodiamond/poly (lactic acid) nanocomposites: effect of nanodiamond on structure and properties of poly (lactic acid)," Composites Part B: Engineering, vol. 41, no. 8, pp. 646-653, 2010.

[12] N. F. Attia, J. P. Rao, and K. E. Geckeler, "Nanodiamondpolymer nanoparticle composites and their thin films," Journal of Nanoparticle Research, vol. 16, article 2361, 2014.

[13] V. N. Mochalin, O. Shenderova, D. Ho, and Y. Gogotsi, “The properties and applications of nanodiamonds," Nature Nanotechnology, vol. 7, no. 1, pp. 11-23, 2012.

[14] Q. Zhang, V. N. Mochalin, I. Neitzel et al., "Fluorescent PLLA-nanodiamond composites for bone tissue engineering," Biomaterials, vol. 32, no. 1, pp. 87-94, 2011.

[15] L. Li, J. L. Davidson, and C. M. Lukehart, "Surface functionalization of nanodiamond particles via atom transfer radical polymerization," Carbon, vol. 44, no. 11, pp. 2308-2315, 2006.

[16] Y. Liu, Z. Gu, J. L. Margrave, and V. N. Khabashesku, "Functionalization of nanoscale diamond powder: fluoro-, alkyl-, amino-, and amino acid-nanodiamond derivatives," Chemistry of Materials, vol. 16, no. 20, pp. 3924-3930, 2004.

[17] A.-Y. Jee and M. Lee, “Thermal and mechanical properties of alkyl-functionalized nanodiamond composites," Current Applied Physics, vol. 11, no. 5, pp. 1183-1187, 2011.

[18] H. Huang, E. Pierstorff, E. Osawa, and D. Ho, "Proteinmediated assembly of nanodiamond hydrogels into a biocompatible and biofunctional multilayer nanofilm," ACS Nano, vol. 2, no. 2, pp. 203-212, 2008.

[19] F. Ostadhossein, N. Mahmoudi, G. Morales-Cid et al., "Development of chitosan/bacterial cellulose composite films containing nanodiamonds as a potential flexible platform for wound dressing," Materials, vol. 8, no. 9, pp. 6401-6418, 2015.

[20] Q. Zhang, V. N. Mochalin, I. Neitzel et al., "Mechanical properties and biomineralization of multifunctional nanodiamondPLLA composites for bone tissue engineering," Biomaterials, vol. 33, no. 20, pp. 5067-5075, 2012.

[21] K. D. Behler, A. Stravato, V. Mochalin, G. Korneva, G. Yushin, and Y. Gogotsi, "Nanodiamond-polymer composite fibers and coatings," ACS Nano, vol. 3, no. 2, pp. 363-369, 2009.

[22] L. Zhao, W. Kai, Y. He, B. Zhu, and Y. Inoue, "Effect of aging on fractional crystallization of poly(ethylene oxide) component in poly(ethylene oxide)/poly(3-hydroxybutyrate) blends," Journal of Polymer Science, Part B: Polymer Physics, vol. 43, no. 19, pp. 2665-2676, 2005.

[23] S. Morlat and J.-L. Gardette, "Phototransformation of water-soluble polymers. I: photo- and thermooxidation of poly(ethylene oxide) in solid state," Polymer, vol. 42, no. 14, pp. 6071-6079, 2001.

[24] T. N. Abraham, D. Ratna, S. Siengchin, and J. KargerKocsis, "Rheological and thermal properties of poly(ethylene 
oxide)/multiwall carbon nanotube composites," Journal of Applied Polymer Science, vol. 110, no. 4, pp. 2094-2101, 2008.

[25] Y. Seok Song, "Rheological characterization of carbon nanotubes/poly(ethylene oxide) composites," Rheologica Acta, vol. 46, no. 2, pp. 231-238, 2006.

[26] Q. Zhang and L. A. Archer, "Poly(ethylene oxide)/silica nanocomposites: structure and rheology," Langmuir, vol. 18, no. 26, pp. 10435-10442, 2002.

[27] J.-L. Gardette, B. Mailhot, F. Posada, A. Rivaton, and C. Wilhelm, "Photooxidative degradation of polyether-based polymers," Macromolecular Symposia, vol. 143, no. 1, pp. 95-109, 1999.

[28] A. Ammala, A. J. Hill, P. Meakin, S. J. Pas, and T. W. Turney, "Degradation studies of polyolefins incorporating transparent nanoparticulate zinc oxide UV stabilizers," Journal of Nanoparticle Research, vol. 4, no. 1-2, pp. 167-174, 2002.

[29] O. Shenderova, V. Grichko, S. Hens, and J. Walch, "Detonation nanodiamonds as UV radiation filter," Diamond \& Related Materials, vol. 16, no. 12, pp. 2003-2008, 2007. 

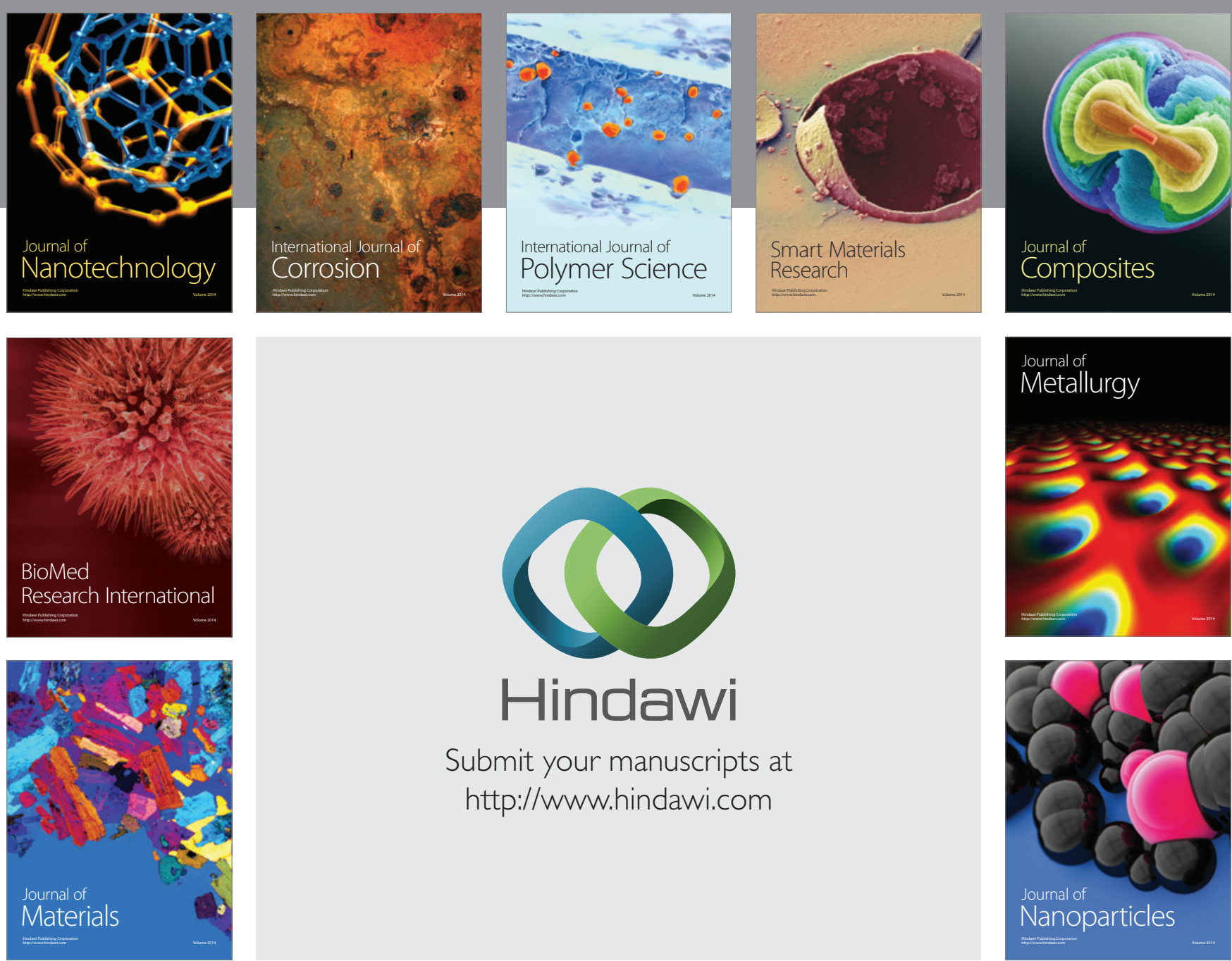

\section{Hindawi}

Submit your manuscripts at

http://www.hindawi.com

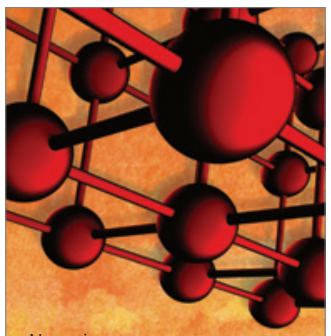

Materials Science and Engineering
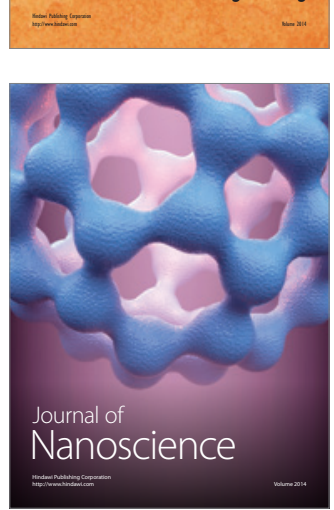
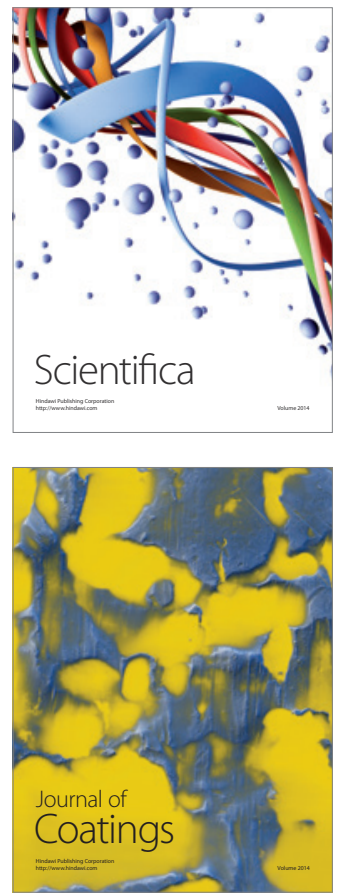
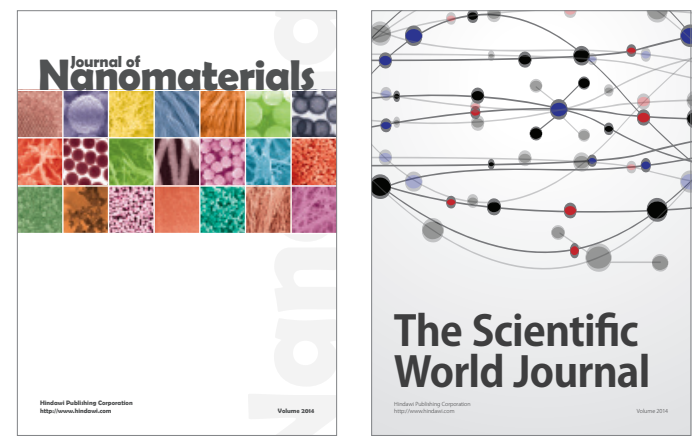

The Scientific World Journal
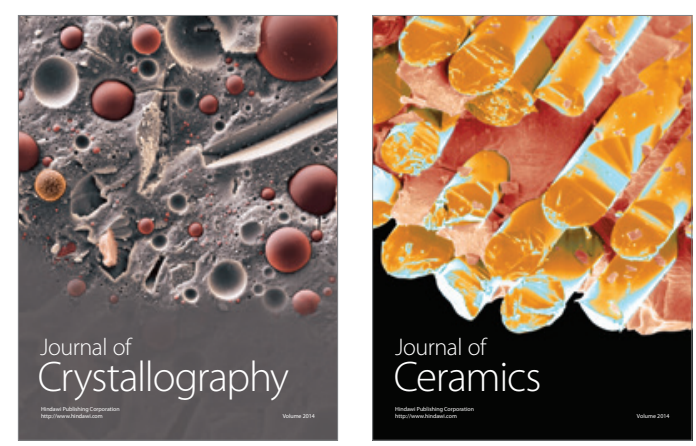
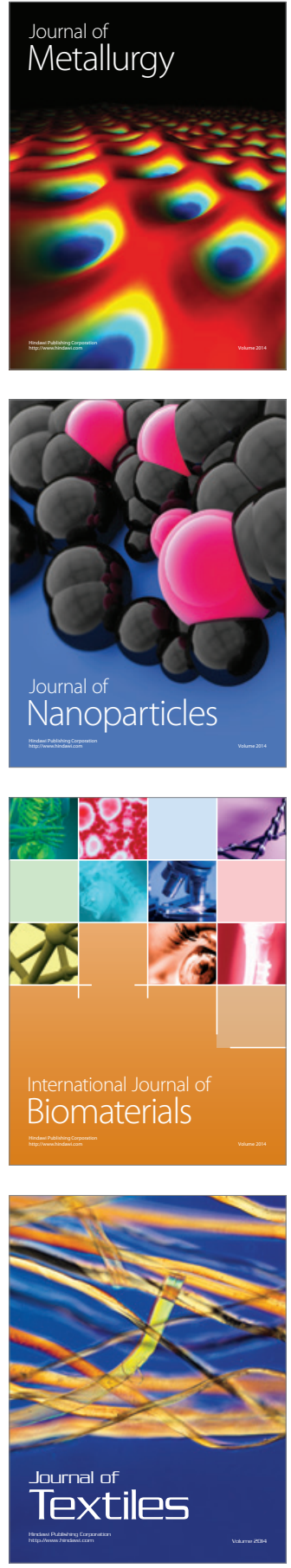